\title{
Timing of surgery after secondary embolic events in infective endocarditis
}

\author{
Sanjeev Bhattacharyya ${ }^{1,2,3}$, Aung Oo ${ }^{2,4}$ \\ ${ }^{1}$ Heart Valve Clinic, St Bartholomew's Hospital, London, UK; ${ }^{2}$ William Harvey Research Institute, Queen Mary University of London, London, \\ UK; ${ }^{3}$ Institute of Cardiovascular Sciences, UCL, London, UK; ${ }^{4}$ Department of Cardiovascular Surgery, St Bartholomew's Hospital, London, UK \\ Correspondence to: Dr. Sanjeev Bhattacharyya, MD, MRCP, FESC. Echocardiography Laboratory \& Heart Valve Clinic, Bart's Heart Centre, St \\ Bartholomew's Hospital, London, UK. Email: Sanjeev.Bhattacharyya@bartshealth.nhs.uk.
}

Submitted Mar 28, 2019. Accepted for publication Apr 17, 2019.

doi: $10.21037 /$ acs.2019.05.02

View this article at: http://dx.doi.org/10.21037/acs.2019.05.02

Infective endocarditis (IE) is one of the most challenging acute cardiovascular pathologies to manage. Embolic complications are common in IE, occurring in between 40$50 \%$ of all cases (1). Manifestations include stroke (embolic, hemorrhagic), peripheral emboli (splenic, renal), pulmonary embolus, coronary artery embolism and vertebral involvement (osteomyelitis). The majority of these occur prior to definitive diagnosis of IE. Unsurprisingly, delays in diagnosis are associated with increased risk of embolic events (2). Treatment with appropriately targeted antibiotics reduces the risk of further embolic events dramatically (3).

Stroke remains a complication associated with significant morbidity and mortality (4). Presentation can range from a silent infarct identified on cerebral imaging, mild motor, sensory or proprioception loss to debilitating deficits an affecting mobility. Cerebral imaging identifies a range of different etiologies from embolic infarct, hemorrhagic stroke to mycotic aneurysm and brain abscess. Each of these has differing size and functional effects. Therefore, management of these patients requires an expanded team to those usually associated with the endocarditis and may include stroke physicians, neurosurgeons and neuroradiologists.

Surgery in patients with IE is recommended in patients with severe valve regurgitation causing heart failure, as well as uncontrolled sepsis despite antibiotics, such as those with aortic root abscess, ongoing fever, or recurrent embolization events despite appropriate antibiotics (5). However, in patients who have had embolic complications, careful assessment of risk and benefits is required. Surgery requiring cardiopulmonary bypass requires use of large doses of anti-coagulation, commonly heparin. This raises concerns regarding the hemorrhagic transformation of ischaemic stroke or worsening of bleeding in those with intra-cerebral hemorrhage.

Surgery after hemorrhagic stroke is associated with a mortality high risk. García-Cabrera et al. (5) found the risk of new cerebral bleeding reduces as the time interval between the index hemorrhage and surgery increases. In this series, $50 \%, 33 \%$ and $20 \%$ patients had worsening intra-cerebral bleeding if surgery was undertaken at $<2$, 2-3 and $>3$ weeks respectively. Okita et al. (6) found in a series of patients with IE and intracranial haemorrhage, patients whose surgery was performed after 7 days had a lower mortality compared to those patients where surgery was undertaken within 7 days of diagnosis. Therefore, the interval between intracerebral bleed and cardiac surgery should be delayed for as long as possible. The European Society of Cardiology guidelines (4) recommend waiting one month after cerebral hemorrhage before surgery. In certain circumstances, a clinical scenario, such as worsening sepsis and hemodynamic instability, may necessitate earlier consideration of surgery. In our practice, we recommend serial magnetic resonance imaging to assess changes/ reduction in the degree of intra-cerebral bleeding to help guiding timing.

The risk of surgery after ischaemic stroke depends on the size and severity of stroke. García-Cabrera et al. (5) found patients with small ischaemic stroke had a higher risk of exacerbation of neurological symptoms and bleeding than those patients without stroke undergoing surgery. The percentage of bleeding events and exacerbation of 
neurological symptoms in patients undergoing cardiac surgery was $11 \%$ in the first week, $10 \%$ in the second week, and $27 \%$ in the third week after surgery. In patients with moderate/severe ischaemic burden, the same group found a high mortality in patients undergoing surgery. However, it is difficult to draw firm conclusions as these were retrospective studies with selection bias, as only $28 \%$ of those with moderate/severe ischaemic stroke underwent surgery. In patients with IE and neurological events, the European Society of Cardiology guidelines recommend (4) surgery can be performed without delay if indicated in patients who suffer from silent embolism or transient ischaemic attack (class 1, level of evidence B). In patients after stroke, they recommend surgery should be considered as long as cerebral hemorrhage has been excluded and the patient is not comatose.

Ultimately, the decision of whether to perform and the timing of cardiac surgery in patients with IE with neurological events will depend on the evaluation of the likely outcomes of IE with and without surgery in the context of the severity of neurological deficit, extent and type of cerebral infarct, taking into account the patient's wishes, co-morbidities and guideline recommendations.

Peripheral embolic events to the abdominal organs (liver, spleen or renal infarcts) or musculoskeletal involvement (discitis, osteomyelitis) in general do not alter the established, guideline recommended indications or timing of cardiac surgery in IE. However, the presence of abscess in these locations requires careful evaluation. The number, size, location, response to therapy and potential for drainage need to be considered. Abscesses not responding to antibiotics (based on systemic features, blood markers and serial imaging) may require drainage. The preference for whether drainage occurs prior to-, or after cardiac surgery requires an individual patient decision depending on the urgency and type of cardiac surgery (mechanical or bioprosthetic prosthesis), hemodynamic status of the patient and mode of abscess drainage (percutaneous or surgical). Drainage may be preferred prior to cardiac surgery to avoid interruption of anti-coagulation post-operatively in those patients requiring mechanical valves or to prevent the risk of infection of a newly implanted valve; however, there is no data to support this.

Coronary embolic events are rare. In a series of 1210 patient with endocarditis, Roux et al. found only $26(2.2 \%)$ patients had an acute coronary syndrome (7). The majority of cases $(88 \%)$ were due to coronary embolism. There is no specific data examining the risk of cardiac surgery after acute coronary syndromes in IE. However, analysis of coronary bypass mortality after acute coronary syndromes has shown no significant difference in outcomes of surgery performed within 48 hours, compared to those performed after 48 hours (8). Therefore, in patients with an indication for surgery in IE, we believe there should be no significant delay in patients with embolic coronary events.

Although guidelines provide a framework for decision making in IE, each patient has unique circumstances, including age, co-morbidities, valve destruction/dysfunction, response to antibiotics and embolic complications. An individualized management plan taking account of these factors within the context guidelines and expertise of a multi-disciplinary team is required.

\section{Acknowledgments}

None.

\section{Footnote}

Conflicts of Interest: The authors have no conflicts of interest to declare.

\section{References}

1. Hubert S, Thuny F, Resseguier N, et al. Prediction of symptomatic embolism in infective endocarditis: construction and validation of a risk calculator in a multicenter cohort. J Am Coll Cardiol 2013;62:1384-92.

2. Young WJ, Jeffery DA, Hua A, et al. Echocardiography in Patients With Infective Endocarditis and the Impact of Diagnostic Delays on Clinical Outcomes. Am J Cardiol 2018;122:650-5.

3. Thuny F, Avierinos JF, Tribouilloy C, et al. Impact of cerebrovascular complications on mortality and neurologic outcome during infective endocarditis: a prospective multicentre study. Eur Heart J 2007;28:1155-61.

4. Habib G, Lancellotti P, Antunes MJ, et al. 2015 ESC Guidelines for the management of infective endocarditis: The Task Force for the Management of Infective Endocarditis of the European Society of Cardiology (ESC). Endorsed by: European Association for Cardio-Thoracic Surgery (EACTS), the European Association of Nuclear Medicine (EANM). Eur Heart J 2015;36:3075-128.

5. García-Cabrera E, Fernández-Hidalgo N, Almirante B, et al. Neurological complications of infective endocarditis: risk factors, outcome, and impact of cardiac 
surgery: a multicentre observational study. Circulation 2013;127:2272-84.

6. Okita Y, Minakata K, Yasuno S, et al. Optimal timing of surgery for active infective endocarditis with cerebral complications: a Japanese multicentre study. Eur J Cardiothorac Surg 2016;50:374-82.

7. Roux V, Salaun E, Tribouilloy C, et al. Coronary events complicating infective endocarditis. Heart

Cite this article as: Bhattacharyya S, Oo A. Timing of surgery after secondary embolic events in infective endocarditis. Ann Cardiothorac Surg 2019;8(6):688-690. doi: 10.21037/ acs.2019.05.02
2017;103:1906-10.

8. Parikh SV, de Lemos JA, Jessen ME, et al. Timing of inhospital coronary artery bypass graft surgery for non-STsegment elevation myocardial infarction patients results from the National Cardiovascular Data Registry ACTION Registry-GWTG (Acute Coronary Treatment and Intervention Outcomes Network Registry-Get With The Guidelines). JACC Cardiovasc Interv 2010;3:419-27. 